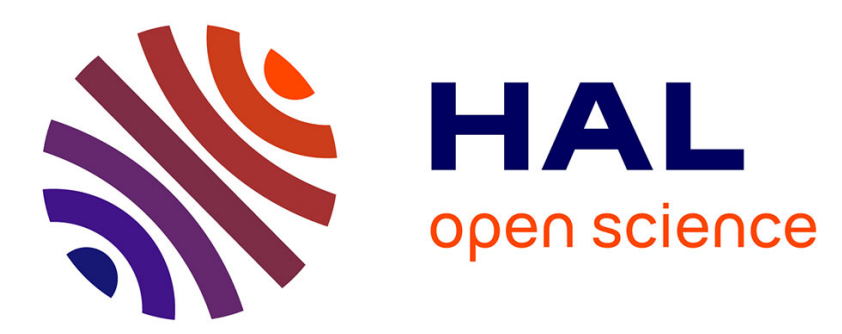

\title{
Homogenization of Dentin Elastic Properties Based on Microstructure Characterization, Statistical Back-Analysis, and FEM Simulation
}

Romain Jeanneret, C. Arson, Elsa Vennat

\section{- To cite this version:}

Romain Jeanneret, C. Arson, Elsa Vennat. Homogenization of Dentin Elastic Properties Based on Microstructure Characterization, Statistical Back-Analysis, and FEM Simulation. 6th Biot Conference on Poromechanics, Jul 2017, Paris, France. pp.1339-1346, 10.1061/9780784480779.166 . hal02458649

\section{HAL Id: hal-02458649}

https://hal-centralesupelec.archives-ouvertes.fr/hal-02458649

Submitted on 7 Mar 2020

HAL is a multi-disciplinary open access archive for the deposit and dissemination of scientific research documents, whether they are published or not. The documents may come from teaching and research institutions in France or abroad, or from public or private research centers.
L'archive ouverte pluridisciplinaire HAL, est destinée au dépôt et à la diffusion de documents scientifiques de niveau recherche, publiés ou non, émanant des établissements d'enseignement et de recherche français ou étrangers, des laboratoires publics ou privés. 


\title{
Homogenization of dentin elastic properties based on microstructure characterization, statistical back-analysis and FEM simulation
}

\author{
January 3, 2017
}

\author{
R. Jeanneret ${ }^{1,2}$, C. Arson ${ }^{1}$, E. Vennat ${ }^{2}$ \\ ${ }^{1}$ Georgia Institue of Technologies, School of Civil and Environnemental engineering, \\ 790 Atlantic Drive, Atlanta, GA, 30318, USA \\ ${ }^{2}$ Laboratoire MSSMat, CNRS, Centrale-Supélec, Université Paris-Saclay, 92290 \\ Châtenay-Malabry, France \\ e-mail : romain.jeanneret_dit_grosjean@centraliens.net
}

\begin{abstract}
The dentinal tissue is made of tubules surrounded by peri-tubular dentin (PTD), embedded in a matrix of inter-tubular dentin (ITD). Hashin and Rosen found exact stiffness bounds for hexagonal patterns of hollow fibers. But Stateof-the-Art micro-macro models rely on simplified microstructure representations and lack experimental validation. The Poissons ratios of dentin microstructure components cannot be determined by direct experimental methods. By contrast, we apply Hashins homogenization scheme to a non-uniform PTD distribution, determined from image analysis. According to Finite Element simulations, a cube containing 60 tubules is a Representative Elementary Volume. Microscopy, nanoindentation and Resonant Ultrasound Spectroscopy data were collected from each dentin sample studied for model calibration. Despite the high variability of microstructure descriptors and mechanical properties, statistical analyses show that Hashins bounds converge and that the proposed model can be used for backcalculating the microscopic mechanical properties of dentin constituents.
\end{abstract}

\section{Introduction}

Dentine is the tissue of the tooth that is located between the pulp and the enamel. Most materials used to repair dentin cavities (e.g., amalgam, cement, sealants) do not last more than 15 years, which raised interest in the micro-macro mechnical modeling of 
dentin [7]. Dentin can be seen as a bundle of hollow fibers of peri-tubular dentin (PTD) embedded in a matrix of inter-tubular dentin (ITD). The rigorous calculation of the stiffness coefficients of a solid with hollow cylinder inclusions remains a challenge. Hashin and Rosen [2] computed exact bounds in the case of a hexagonal pattern of fibers, assuming a transverse isotropy for the homogenized material. Improvements were then made by Herve and Zaoui [3], Hongjun [4], Shi [12] and Tsukrov [11]. Due to their complexity, these models were never used to back analyze the mechanical properties of dentin constituents. Previous studies of dentin homogenized properties were based on Reuss and Voigt bounds [5], the self-consistent method [6], averaging techniques assuming periodic structure [1] and Hashin and Rosen bounds [9]. However, none of these models was validated experimentally and the values of the microscopic Poisson's ratios had to be postulated instead of being calibrated. By contrast, we establish a stiffness homogenization scheme based on microstructure images, nano-indentation test results and measured macroscopic mechanical properties. In the first section, we explain the dentin stiffness model, which is based on Hashin's homogenization scheme with a non-uniform distribution of peri-tubular dentin volume around the tubules, determined from image analysis. In the second section, we present a calibration technique that can be employed for tooth dentinal tissue.

\section{Micro-Macro Model for Dentin Stiffness}

\subsection{Homogenization Scheme}

Figure 1 shows a Scattered Electron Microscope (SEM) image of dentin, taken in a horizontal plane in a tooth. Inter-Tubular Dentin (ITD), made of collagen and apatite, forms a matrix that contains fluid-filled tubules surrounded by Peri-Tubular Dentin (PTD), mostly made of apatite [8]. Tubules are between $2.9 \mu \mathrm{m}$ and $3.02 \mu \mathrm{m}$ in size and the tubule concentration is between 18.2 fibers $/ \mathrm{mm}^{2}$ and $24.162 \mathrm{fibers} / \mathrm{mm}^{2}$ [10]. The surface concentration of tubules is higher close to the pulp than to the enamel, because tubules spread out forming a fan. In the following, we propose a homogenization scheme for an elementary block of dentin, at the scale of which tubules can be consisdered parallel (Fig.2). Accordingly, we assume that dentine follows a transversely isotropic behavior, characterized by five elastic parameters.

In the following, we consider an elementary volume of composite material containing parallel hollow cylinders oriented along direction 1 . When cylinders are periodically distributed according to a hexagonal pattern, the lower and upper bounds of the five independent elastic parameters $E_{1}, E_{2}, G_{12}, \nu_{12}$ and $G_{23}$ are equal [2]. For a random distribution, only the transverse shear modulus $G_{23}$ has two distinct bounds. In the original model of Hashin and Rosen, hollow cylinders are aligned and have a circular cross section characterized by the same ratio inner radius / outer radius (noted 

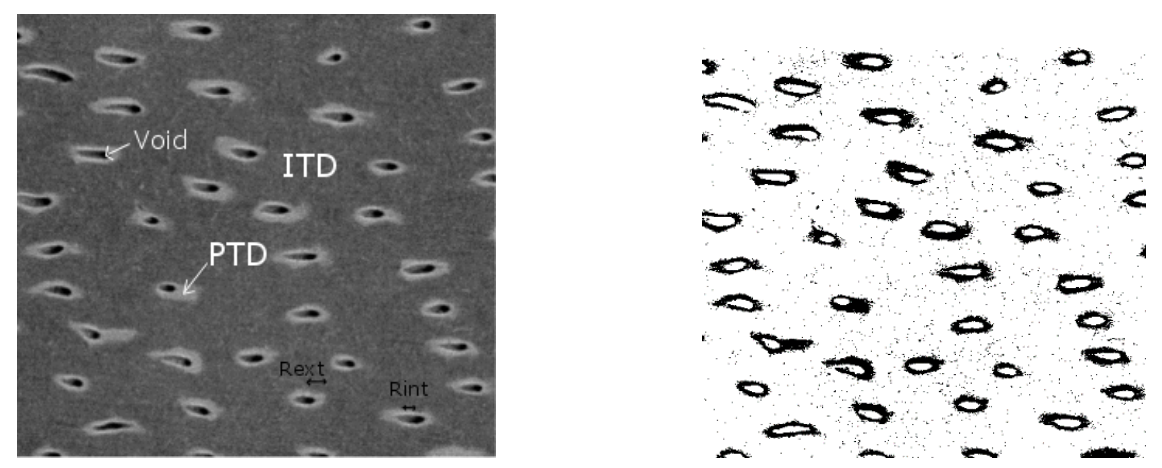

Figure 1: SEM image of dentin (left) taken in a horizontal plane of the tooth. Binarized image (right), showing the PTD in black.

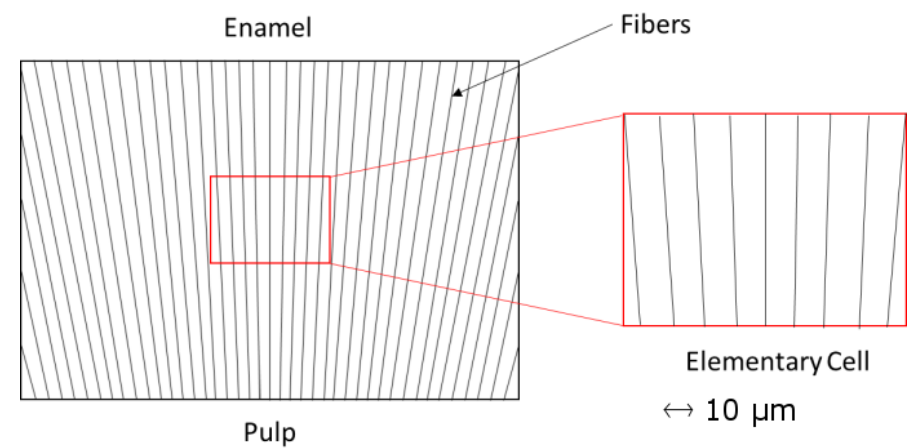

Figure 2: Schematic view of the elementary block of dentin modeled with the proposed homogenization scheme. Tubules are considered parallel, which allows assuming transverse isotropy.

$\alpha$ ). In this work, the non-uniform distribution of $\alpha$ is modeled by a Gaussian law:

$$
\rho(\alpha)=\frac{1}{\sqrt{2 \pi} \sigma} \cdot e^{-\frac{\left(\mu_{\alpha}-\alpha\right)^{2}}{2 \sigma_{\alpha}^{2}}}
$$

In which $\mu_{\alpha}$ and $\sigma_{\alpha}$ are the mean and standard deviation of the probability density function $\rho(\alpha)$, respectively. Considering that in dentine, $\alpha$ varie sbetween 0.2 and 0.8 , for any of the homogenized elastic moduli $C_{i j}(\alpha)$, Reuss and Voigt bounds are computed as follows:

$$
\int_{\alpha=0.2}^{\alpha=0.8} \rho(\alpha) C_{i, j}(\alpha) d \alpha \leq \overline{C_{i j}} \leq \int_{\alpha=0.2}^{\alpha=0.8} \rho(\alpha) C_{i, j}^{-1}(\alpha) d \alpha
$$

where $C_{i, j}(\alpha)$ is the homogenized stiffness coefficient for a given value of $\alpha$, and $\overline{C_{i j}}$ our global homogenized stiffness coefficient. The proposed homogenization scheme requires seven microscopic parameters: the Young's modulus and Poisson's ratio of the ITD and of the PTD, statistical parameters $\mu_{\alpha}$ and $\sigma_{\alpha}$, and the volume fraction of ITD in the elementary cell (noted $v_{m}$ ). 

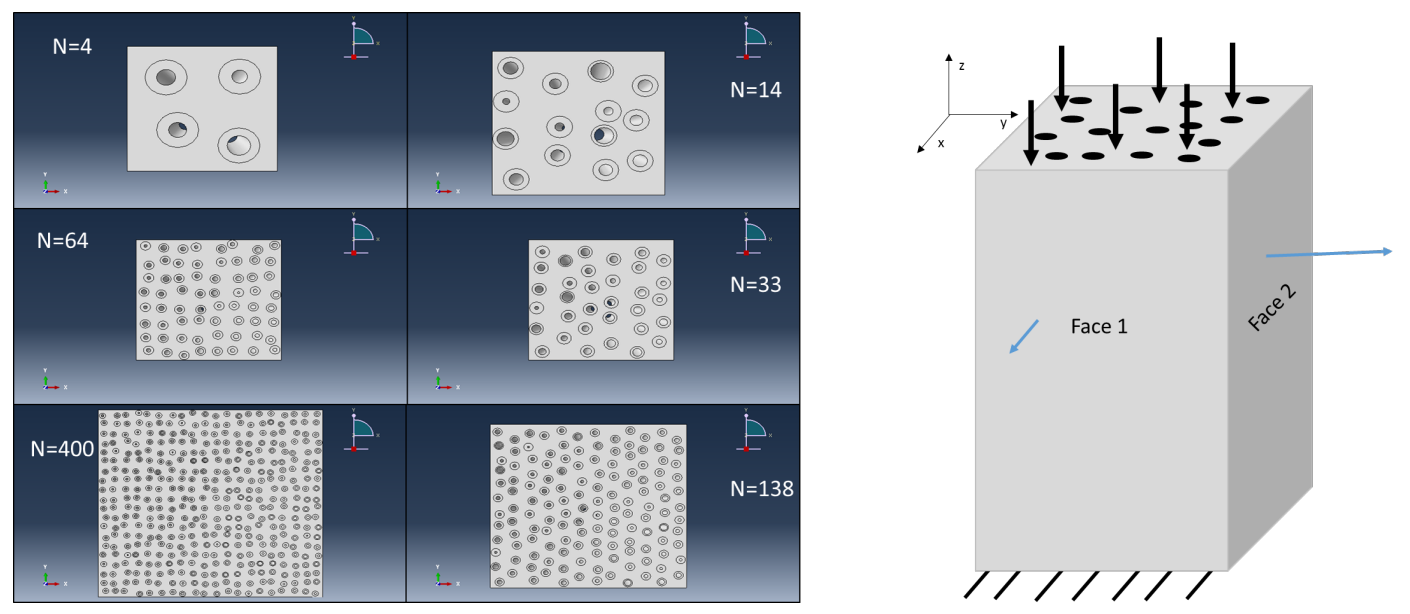

Figure 3: FEM models used to simulate strain-controlled compression tests. The load was applied along the $\mathrm{x}$-axis (direction of the tubules) and the transversal displacements on orthogonal faces were calculated.

\subsection{Representative Elementary Volume (REV)}

We used the Finite Element Method (FEM) to simulate the mechanical response of dentin subjected to a strain-controlled uniaxial compression along the axis of the tubules. Numerical samples were parallelepipedic with 4 to 400 parallel hollow cylinders of circular section and same external radius. Cylinders are assigned a random distribution of $\alpha$, which determines the inner radius distribution. Tubules were placed on rectangular and hexagonal grids, with a random distribution of offsets along the $\mathrm{x}$ and $\mathrm{y}$ directions. In total, 20 FEM models were tested for each of the two patterns (Figure 3). Results for hexagonal and rectangular pattern are plotted in Figure 4. The difference between transverse displacements on opposite vertical faces decreases with the number of cylinders, and then stabilizes at around $2 \%$ for samples with 60 cylinders or more, for both hexagonal and rectangular patterns. This means that the assumption of transverse isotropy is valid for dentin samples containing 60 tubules or more, which we consider as the Representative Elementary Volume (REV).

\section{Model Calibration}

\subsection{Experimental Data}

We calibrate the microscopic Poisson's ratios of ITD and PTD by fitting the macroscopic elastic parameters of dentin against measures obtained by Resonant Ultrasound Spectroscopy (RUS) at LIB (Paris VI University), with constrained values for the four other microscopic parameters. The Young's moduli of ITD and PTD were obtained by nano-indentation at MSSMat (Ecole Centrale de Paris). ESEM microstructure images obtained at LMS (Ecole Polytechnique), which allowed determining the probability 

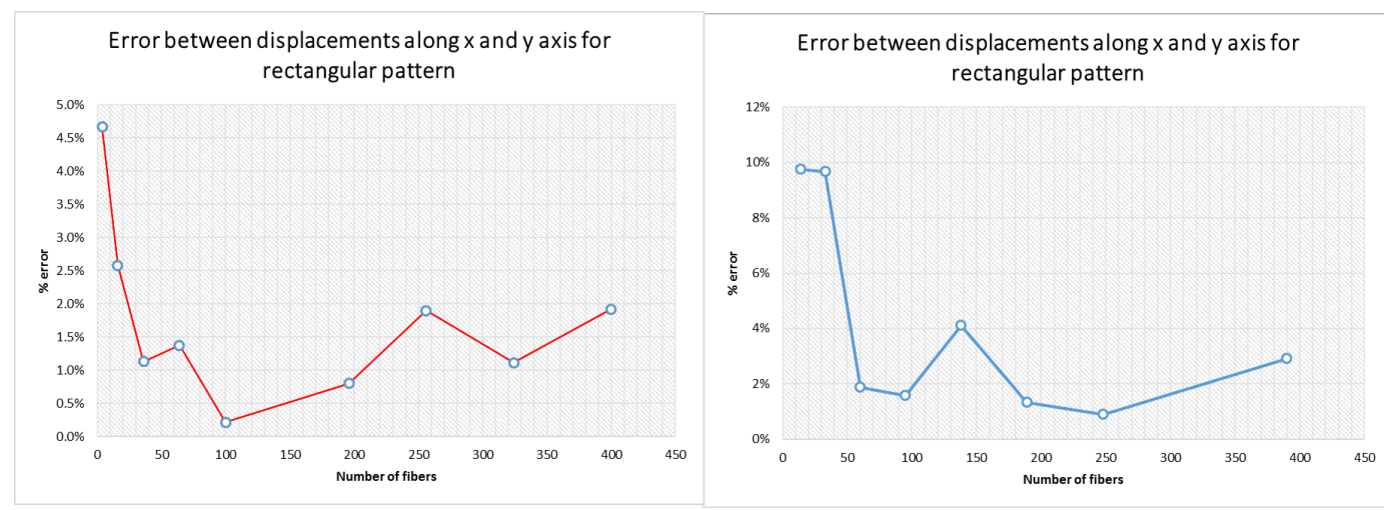

Figure 4: Difference between transversal displacements on opposite vertical faces of the FEM models shown in Figure 3. A small error on the displacements indicates that the assumption of transverse isotropy is valid, and that the FEM domain is a REV.

density function (pdf) of the ratio $\alpha$ and the volume fraction of ITD $\left(v_{m}\right)$. RUS and nano-indentation results are summarized in Table 1, and results of image analysis are presented in Table 2. Note that RUS, nano-indentation and imaging were performed on the same samples, which ensured consistency between the measures used for calibration. To determine the pdf of $\alpha$, we obseved 1,186 fibers in 6 SEM images. We modeled the pdf as a truncated Gaussian distribution, varying between 0.2 and 0.8 . The averaged error between the pdf obtained by image analysis and the fitted pdf is $15 \%$, which was considered acceptable, provided the variability of biological parameters. To find $v_{m}$, we analyzed 24 images of dentin in cross-sections located at different elevations in the tooth. As explained above, the large difference of tubule surface density between sections close to the pulp and section close to the enamel results in a great variance. In the following, we take the average value of $v_{m}$ for the calculations.

Table 1: Dentin mechanical properties obtained by RUS and nanoindentation

\begin{tabular}{lllllll}
\hline & \multicolumn{3}{c}{ Resonant ultrasound spectroscopy } & \multicolumn{2}{c}{ Nanoindentation } \\
\hline$E_{1}(\mathrm{GPa})$ & $E_{2}(\mathrm{GPa})$ & $\nu_{12}$ & $\nu_{23}$ & $G_{12}(\mathrm{GPa})$ & $E_{I T D}(\mathrm{GPa})$ & $E_{P T D}(\mathrm{GPa})$ \\
\hline 23.35 & 21.9 & 0.298 & 0.512 & 9.63 & 18.46 & 31.08 \\
\hline
\end{tabular}

Table 2: Results of dentine image analysis. SD: Standard Deviation.

\begin{tabular}{llll|llll}
\hline \multicolumn{3}{c|}{$\alpha$ determination } & \multicolumn{4}{c}{$v_{m}$ determination } \\
\hline Average & SD & Min & Max & Average & SD & Min & Max \\
\hline 0.49 & 0.093 & 0.15 & 0.79 & 0.75 & 0.0754 & 0.60 & 0.86 \\
\hline
\end{tabular}




\subsection{Convergence of Hashin's Bounds}

As explained above, Hashin's bounds are equal for $E_{1}, E_{2} ; G_{12}$ and $\nu_{12}$. In order to check the suitability of our model to homogenize dentin properties, we check that the bounds of the transverse shear modulus $G_{23}$ converge for the range of values taken by the seven microstructure parameters found for dentin. We use a genetic algorithm to find the maximum difference between the two bounds of $G_{23}$ (noted $\Delta_{G 23}^{\max }$ ) under the following constraints: the Young's moduli of the ITD and PTD are assigned the values of $E_{I T D}$ and $E_{P T D}$ found by nano-indentation (Table 1); the Poisson's ratios of ITD and PTD vary between 0 and $0.5 ; v_{m}$ ranges between 0.6 and 0.86 , according to image analyses (Table 2); and we divide the range of values taken by $\alpha$ into seven intervals between 0.2 and 0.8 . We calculate then the maximum difference beween the two bounds of the transverse shear modulus on that interval (noted $E_{i}$ for the i-th interval). The average maximum difference between the two bounds is calculated as:

$$
\Delta_{G 23}^{\max }=\int_{0.2}^{0.8} \rho(\alpha) \times e(\alpha) d \alpha, \quad e(\alpha)=E_{i} i n\left[\alpha_{i}^{\min } ; \alpha_{i}^{\max }\right]
$$

In which $\left[a_{i}^{\text {min }} ; a_{i}^{\max }\right]$ designates the $i^{\text {th }}$ of the seven subintervals used for the discretized calculation of the bounds difference. We tested the optimization techniques for four ranges of values $\left[a_{1}^{\min } ; a_{7}^{\max }\right]$ (and adapted the seven subintervas accordingly). The corresponding values of $E$ are provided in Table 3. The global upper bound for $\Delta_{G 23}^{\max }$ is equal to $24.1 \%$. Note that the values of the Poisson's ratios were fixed on each interval, and that the discretization was coarse (only seven intervals). Thus the optimization method employed in this study overpredicts errors. A maximum difference of $24.1 \%$ in the bounds of the shear modulus is considered acceptable.

Table 3: Maximum difference between the bounds of hte transverse shear modulus for difference ranges of values of $\alpha$

\begin{tabular}{lllll}
\hline Interval for $\alpha$ & {$[0.2 ; 0.8]$} & {$[0.3 ; 0.7]$} & {$[0.4 ; 0.6]$} & {$[0.45 ; 0.55]$} \\
\hline$E_{i}(\%)$ & 79 & 41 & 19 & 13 \\
\hline
\end{tabular}

\subsection{Back calculation of microscopic Poisson's ratio}

We now calibrate the microscopic Poisson's ratios of ITD and PTD $\left(\nu_{I T D}\right.$ and $\left.\nu_{P T D}\right)$ by fitting the macroscopic elastic parameters of dentin against measures obtained by RUS, under the constraint that $E_{I T D}, E_{P T D}, \mu_{v_{m}}, \mu_{\alpha}$ and $\sigma_{\alpha}$ take the values determined by nano-indentation and image analysis (Tables 1 and 2). Using * and Hashin superscripts to refer to RUS measures and model predictions respectively, we define the following 
function:

$$
\begin{aligned}
& F_{1}\left(\nu_{I T D}, \nu_{P T D}\right)=\frac{1}{7}\left[\sum_{\Gamma} \Delta_{\Gamma}+\delta_{G 23}+\delta_{\text {stat }}\right] \\
& \Delta_{\Gamma}=\frac{\left|\Gamma^{\operatorname{Hashin}}\left(\nu_{I T D}, \nu_{P T D}\right)-\Gamma^{*}\right|}{\Gamma^{*}}, \quad \Gamma=E_{1}, E_{2}, \nu_{12}, G_{12}, G_{23} \\
& \delta_{\text {stat }}=\frac{\max \left(\Delta_{\Gamma}\right)-\min \left(\Delta_{\Gamma}\right)}{\max \left(\Delta_{\Gamma}\right)}, \quad \delta_{G 23}=\frac{G_{23}^{\max }-G_{23}^{\min }}{G_{23}^{\min }}
\end{aligned}
$$

Since the transverse shear modulus is characterized by two bounds only, $\Delta_{G_{23}}$ is taken equal to the relative error between $G_{23} *$ and the closest bound, and to 0 if to 0 if $G_{23} *$ is outside of the bounds found numerically. The parameter $\delta_{\text {stat }}$ is used to balance the optimization criteria between the five macroscopic elastic properties, and $\delta_{G 23}$ is introduced to put more weight on the solutions for which the two bounds of the shear modulus are close. We used a genetic algortihm to optimize values of the ITD and PTD Poisson's ratios in the range $[0.05 ; 0.5]$. We found $\nu_{I T D}=0.381$ and $\nu_{P D T}=0.344$. The corresponding value of the fitness function was $18 \%$ and the maximum difference between the two bounds of the transverse shear modulus was $0.23 \%$. The relative errors for the macroscopic elastic parameters were the following: $\Delta_{E_{1}}=14 \% ; \Delta_{E_{2}}=14 \%$; $\Delta_{\nu_{12}}=13 \% ; \Delta_{G_{12}}=26 \% ; \Delta_{G_{23}}=22 \%$.

\section{Conclusions}

Restorative materials used to fill teeth cavities are anchored in dentin. State-of-the-Art micro-macro models rely on simplified microstructure representations and lack experimental validation. The Poissons ratios of dentin microstructure components cannot be determined by direct experimental methods. We model dentin as a bundle of parallel hollow cylinders made of tubules surrounded by Peri-Tubular Dentin (PTD), embedded in a matrix of Inter-Tubular Dentin (ITD). By contrast with the previous models, we establish a homogenization scheme for a non-uniform distribution of PTD, determined from image analysis. The minimum size of the Representative Elmentary Volume is a cube containing 60 tubules. Microscopy, nano-indentation and Resonant Ultrasound Spectroscopy data were collected from each dentin sample studied for model calibration, which is unprecedented. Statistical analyses show that Hashins bounds converge and that the proposed model can be used for back-calculating the microscopic mechanical properties of dentin constituents, in particular the most probable values of the Poisson's ratios of ITD and PTD. More experimental results will be collected to validate the model and study damaged or restored tissues.

\section{References}

[1] B. BAR-ON AND H. D. WAGNER, Elastic modulus of hard tissues, Journal of Biomechanics, 45 (2012), pp. 672-678. 
[2] Z. Hashin AND B. W. Rosen, The elastic moduli of fiber-reinforced materials, Journal of Applied Mechanics, (1964), pp. 223-235.

[3] E. HERVE AND A. ZAOUI, n-layered inclusion-based micromechanical modelling, International Journal of Engineering Science, (1993).

[4] X. Honguun, S. Zhifei, And Z. TaOtaO, Elastic analyses of heterogeneous hollow cylinders, Mechanics Research Communications, 33 (2006), pp. 681-691.

[5] J. KATZ, Hard tissue as angle composite material - i. bounds on the elastic behavior, Journal Biomechanics, 4 (1971), pp. 455-473.

[6] J. Kinney, M. Balooch, G. Marshall, and S. Marshall, A micromechanics model of the elastic properties of human dentine, Archives of Oral Biology, 44 (1999), pp. 813-822.

[7] C. Lloyd AND S. S. ET AL., Dental materials : 1993 literature review, Journal of Dentistry, 23 (1995), pp. 67-93.

[8] G. W. Marshall, S. J. Marshall, J. H. Kinney, and M. Balooch, The dentine substrate : structure and properties related to bonding, Journal of Dentistry, 25 (1997), pp. 441-458.

[9] Q.-H. QIN AND M. SWAIN, A micro-mechanics model of dentin mechanical properties, Biomaterials, 25 (2004), pp. 5081-5090.

[10] R. Schilke, J. A. Lisson, O. Bauss, and W. Geurtsen, Comparison of teh number and diameter of dedentin tubules in human and bovine dentine buy scanning electron microscopic investigation, Archives of oral Biology, 45 (2000), pp. 355-361.

[11] I. TSUKROV AND B. DRACH, Elastic deformation of composite cylinders with cylindrical orthotropic layers, Solids and Structures, 47 (2010), pp. 25-33.

[12] S. Zhifei, Z. TaOtaO, and X. Honguun, Exact solutions of heterogeneous elastic hollow cylinders, Composite S, 79 (2007), pp. 140-147. 